\title{
Study on the Ability and Stability of Multi-fingered Hands Robot for Grasping Explosives
}

\author{
Haijun Mo ${ }^{\mathrm{a}}$, Zhisheng Lin ${ }^{\mathrm{b}}$, Jiajun Zhou ${ }^{\mathrm{c}}$ \\ South China University of Technology, School of \\ Mechanical Engineering, Guangzhou, China
}

amohj@scut.edu.cn, b824414996@qq.com, c929191729@qq.com

\begin{abstract}
The posture of the explosive disposal robot is changing constantly when it is working or carrying the explosives. The change of the posture results in the change of the loading direction and finally influences the grasping ability of multi-fingered hands. This paper focuses on the ability of the explosive disposal robot for grasping explosive in any posture so as to seek for the best one and analyze the relation between the different postures and the weight of the explosive. Meanwhile, it studies on the stability of the explosive grasped by the multi-fingered hands under the static and dynamic impact load and analyze the minimal normal force of the robot for steadily grasping under the dynamic impact load. Compared with the bearable maximal normal force of the explosive, it judges the stability and improves the safety and reliability of operation.
\end{abstract}

Keywords-explosive; multi-fingered hands; grasping ability; stability.

\section{INTRODUCTION}

By using the multi-fingered hands for grasping the explosives in the operation, the operating flexibility and reliability of the robot can be improved. The posture of the explosive disposal robot is changing all the time when it is working or transporting the explosives, then it will cause the variation in the forced direction of the explosives and ultimately affected the grasping ability of multi-fingered hands. Therefore, it is full of practical significance to study the maximal grasping weight of the explosives in any position. At first, this paper carries out the study that how much weight of the explosives could be caught by the explosive disposal robot in any position, then it analyzes the relation between the different postures of the robots and the grasping weight of the explosives so as to seek for the best posture for grasping. At the same time, it studies the grasping ability under the constraints of maximal contact force.

Besides, in order to make sure of the grasping stability of the explosives under the impact load and avoid them falling off, which is caused by the vibrates of the explosive disposal robot, no only the maximal contact force but also the stability of the explosive, which is under the dynamic load, should be considered in operation. This paper focuses on the minimal normal contact force of the explosive disposal robot, which can grasp the explosive steadily under the dynamic load, then it compares with the affordable maximal contact force of the explosives and judges the grasping stability so as to avoid them falling off, finally the safety and reliability of the operation are improved.

\section{THE ANALYSIS OF THE CAPACITY FOR THE ROBOT TO GRASP THE EXPLOSIVES}

When the grasping position of explosive is given, the maximal grasping ability depends on the grasping posture of the explosive disposal robot in the operation. The paper mainly focuses on the study that different grasping postures effect on the grasping ability and stability.

According to the theory of external wrench ${ }^{[1]}$, any force system acting on the rigid body can be combined into concentrated force acting on a certain line and a torque around it. The force and the torque can be collectively referred to external wrench $\boldsymbol{F}_{\text {ext }}$.

According to the research, the maximum grasping ability of the multi-fingered hands distributes evenly in the large circle plane when grasping the sphere explosive ${ }^{[2]}$. Hence, choose the large circle plane as the grasping plane of multi-fingered hands then establish the coordinate system. The posture of the robot is determined by the direction of external wrench in the coordinate system. Assume that there are three fingers in a multi-fingered hand with the frictional point contact between the fingertip and the exlosive, establish the coordinate system of the object $\{\boldsymbol{O}\}$ through the centre of sphere and choose the coordinate axis $\mathrm{x}$ through contact point between the fingertip and the explosive. Shown in Fig 1, the included angle between the external wrench $\boldsymbol{F}_{\text {ext }}$ and the grasping plane is $\alpha$ and the one between axis $x$ and the projection of $\boldsymbol{F}_{\text {ext }}$ on $x O y$ plane is $\beta$. In this way, when the posture of the explosive disposal robot changes, the relation between the external wrench and the angle $\alpha$ and $\beta$ also changes. Based on the objective of maximal grasping explosive, it establishes a mathematic optimal model of the changing relationship, which is between the weight of the explosive and the angle $\alpha$ and $\beta$, and seek for the posture for grasping the maximal and minimal weight of the explosives. 


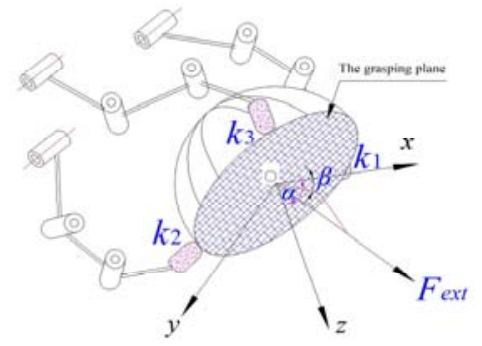

Figure 1. The posture of robot for grasping the explosive

When the grasping object are explosives, the external wrench mapped by the contact force of the fingers can be shown as

$$
G f_{\text {tip }}=-m g
$$

Where $f_{\text {tip }}$ is the force of the object added by the fingertip, or called the contact force which can be presented as

$$
f_{\text {tip }}=\left[\begin{array}{c}
f_{x} \\
f_{y} \\
f_{n}
\end{array}\right]
$$

Above the formula, $\boldsymbol{f}_{\boldsymbol{x}}$ and $\boldsymbol{f}_{\boldsymbol{y}}$, which can be also named as operating force $\boldsymbol{f}_{\mathrm{T}}$, are the tangential forces of the contact point between the finger and the explosive and $f_{\mathrm{n}}$ or $\boldsymbol{f}_{\mathrm{N}}$, is the normal force of the contact point with the direction pointing to the internal surface of the explosive, which are shown in Fig 2.

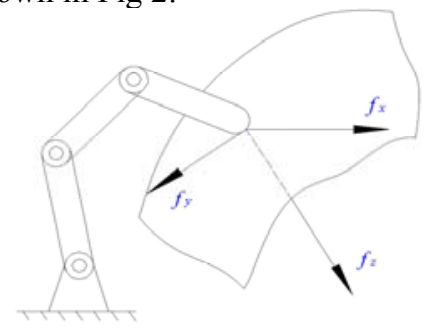

Figure 2. The contact force of multi-fingered hand

Where $\boldsymbol{G}$ is the grasping matrix, which is the map between the contact force of fingers and the external wrench. When the grasping position evenly distributed in the large circle of the sphere, where is best, $\boldsymbol{G}$ can be solved.

Based on the optimization objective min $-m g$, which is the balanceable maximum weight of the exlosive by multi-fingered hands and considering the constraint under the grasping condition ${ }^{[3]}$, it establishes the optimization model.

$$
\begin{cases}\min & -m g \\
\text { s.t. } & G f_{t i p}=-\left[\begin{array}{c}
m g \cos \alpha \cos \beta \\
m g \cos \alpha \sin \beta \\
m g \sin \alpha
\end{array}\right] \\
& -f_{n i} \leq 0 \\
& \sqrt{f_{x i}^{2}+f_{y i}^{2}}-\mu f_{n i} \leq 0 \\
& J^{T} f_{t i p}-\tau_{\max } \leq 0 \\
& -J^{T} f_{t i p}+\tau_{\min } \leq 0\end{cases}
$$

Where $\boldsymbol{J}$ is the jacobian matrix of the mechanism of the robot and $\boldsymbol{\tau}$ is every joint torque.

The neural network model is established and optimized with the input variables of $\alpha$ and $\beta$ and its optimize result is shown in Fig 3.

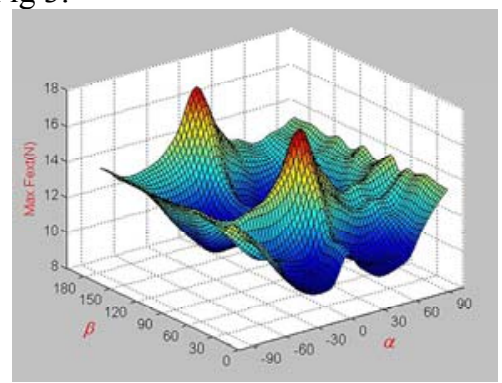

Figure 3. The relationship between the weight of explosive and the posture of robot

It reveals from the figure that the ability of robot for grasping explosive and its posture have something to do with the variables of $\alpha$ and $\beta$, and the maximum weight is located in the grasping plane, i.e. $\alpha=0$, where is in the middle between the finger $k_{1}$ and $k_{2}, k_{2}$ and $k_{3}$ also, $k_{1}$ and $k_{3}$, i.e.

$$
\left\{\begin{array}{l}
\alpha=0 \\
\beta=60^{\circ}
\end{array},\left\{\begin{array}{l}
\alpha=0 \\
\beta=180^{\circ}
\end{array},\left\{\begin{array}{l}
\alpha=0 \\
\beta=300^{\circ}
\end{array} .\right.\right.\right.
$$

How the grasping weight of explosive change over the variation of $\alpha$ when $\beta=60^{\circ}$ is shown in Fig 4, apparently, the grasping ability is best when $\alpha=0$.

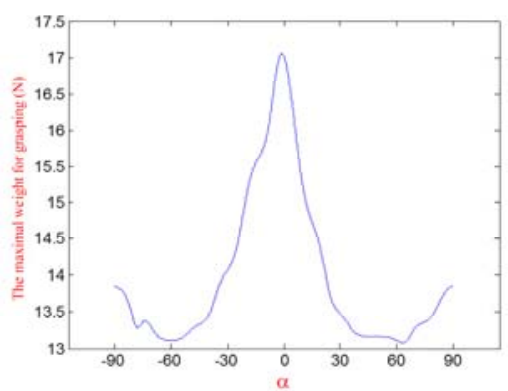

Figure 4. The relationship between the maximal grasping weight and $\alpha$

Additionally, the minimum appears at 


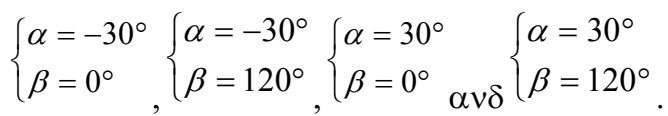

While the explosive disposal robot are conveying the explosives, the position and posture are changing, so it should choose the minimum of the optimization algorithm above when considering the multi-fingered hands to grasp the explosives steadily in any position. Only in this way, it can ensure the grasping steady.

\section{THE ANALYSIS OF THE STEADY OF THE EXPLOSIVE}

\section{A. The Analysis of the Minimal Contact Force for Grasping under the Dynamic Load}

The analysis above is the grasping ability of multi-fingered hand under the static conditions. However, it will result the vibration of the grasping explosive as the explosive disposal robot is carrying or stepping over the barriers and generates dynamic load. Therefore, in order to make sure of the stability to grasp the explosives, the grasping has to meet the force equilibrium condition under the dynamic load.

When the explosive disposal robot is moving and the explosive grabbed by the multi-fingered hand is subjected to impact loads ${ }^{[3]}$, the kinetic equation can be shown in Formula 1.

$$
\boldsymbol{G f}_{\text {tip }}=-\left[\begin{array}{l}
\overrightarrow{\boldsymbol{F}}_{\text {ext }} \\
\tau
\end{array}\right]=-\left[\begin{array}{l}
\boldsymbol{m} \overrightarrow{\boldsymbol{a}}+\boldsymbol{m} \overrightarrow{\boldsymbol{g}} \\
\boldsymbol{I}_{0} \dot{\omega}_{0}+\omega_{0} \times \boldsymbol{I}_{0} \omega_{0}
\end{array}\right]
$$

Where $\boldsymbol{m} \boldsymbol{g}$ is the weight of the explosive, $\boldsymbol{m a}$ is the inertia force, $\boldsymbol{I}_{0}$ is rotary inertia of the explosive, $\boldsymbol{\omega}_{0}$ is the angular velocity, and $\dot{\omega}_{0}$ is the angular acceleration. As the surface of the multi-fingered hands is hard, there is no torque between the explosive and them, so choose $\tau=0$.

The equation of (3) can be divided into two parts ${ }^{[4]}$, i.e.

$$
f_{\text {tip }}=f_{T}+f_{N}=-G^{+} F_{e x t}+N \chi
$$

Where $\boldsymbol{G}^{+}=\boldsymbol{G}^{\boldsymbol{T}}\left(\boldsymbol{G} \boldsymbol{G}^{\boldsymbol{T}}\right)^{-1}$ is the generalized inverse matrix of Moore-Penrose of $\boldsymbol{G}^{[5]}, \boldsymbol{G}^{+} \boldsymbol{F}_{\text {ext }}$ is the particular solution of equation (3), which is the minimum norm solution amount to the contact force balanced with external wrench and called operation force $\boldsymbol{f}_{\mathrm{T}}$.

$N=\left(I-G^{+} G\right) \in R^{n-m}$ is the matrix, which is made up by the null space base vector of grasping matrix $G$. $\left(\boldsymbol{I}-\boldsymbol{G}^{+} \boldsymbol{G}\right) \boldsymbol{\chi}$ is the internal force $\boldsymbol{f}_{\mathrm{N}}$, i.e. the normal force of the finger.

Therefore, in order to make sure of the grasping steadily, there must be some appropriate contact force adding to the object. This contact force between the finger and the object consists of operating force and normal force and the finger has to add the moving internal force to the object so that it can avoid the relative sliding between the finger and the object and no separate ${ }^{[6]}$.

Above the formula, $\chi$ is a freely selectable vector and it determines an infinite number of solutions of the normal force $\boldsymbol{f}_{\boldsymbol{N}}$. How to choose vector $\boldsymbol{\chi}$ has something to do with the magnitude of the contact force ${ }^{[7]}$. The overlarge normal force can not only result the body's wastage and the waste of energy but also the deformation of explosive, especially the brittle and dangerous goods, which may even cause explosion to threaten the surrounding environment and the safety of the robot itself ${ }^{[9]}$. Therefore, based on the grasping steadily, the best method to improve the safety is to obtain the minimum normal force.For getting the minimal normal force, it optimizes the vector $\chi$ and minimizes it, so the calculation of the minimum normal force for grasping with multi-fingered hands is the optimization problem under the given constraints.

\section{B. The Mathematical Model of Minimum Internal Force}

According to the constrains in Formula 1, the linear constraint of grasping with multi-fingered hands is

$$
\left\{\begin{array}{l}
-f_{n i} \leq 0 \\
J^{T} f_{t i p} \leq \tau_{\max } \\
-J^{T} f_{t i p} \leq-\tau_{\min }
\end{array}\right.
$$

Moreover, every normal force of the contact point has to be located in the friction cone and form nonlinear constraints ${ }^{[8]}$ to avoid the relative slip between the fingers and the explosives.

$$
\sqrt{f_{x i}^{2}+f_{y i}^{2}}-\mu f_{z i} \leq 0
$$

After linearizing the nonlinear constraint above, it can be shown in matrix like

$$
\left[\begin{array}{ccc}
-1 & 0 & \mu^{\prime} \\
1 & 0 & \mu^{\prime} \\
0 & -1 & \mu^{\prime} \\
0 & 1 & \mu^{\prime}
\end{array}\right]\left[\begin{array}{l}
f_{x i} \\
f_{y i} \\
f_{z i}
\end{array}\right] \leq\left[\begin{array}{l}
0 \\
0 \\
0 \\
0
\end{array}\right]
$$

Where $\mu^{\prime}$ is the equivalent coefficient of friction and apparently $\mu^{\prime}=\sqrt{2} \mu / 2$.

Making Formula (5) and Formula (6) to the linear inequality constraints based on the variation of contact force $f_{\text {tip }}$, the vector can be shown as 


$$
A f_{\text {tip }} \leq B
$$

Where $m$ is the number of the fingers and $\boldsymbol{A} \in \mathfrak{R}^{7 \boldsymbol{m} \times 3 \boldsymbol{m}}, \boldsymbol{B} \in \mathfrak{R}^{7 \boldsymbol{m} \times 1}, \boldsymbol{f}_{\text {tip }} \in \mathfrak{R}^{3 \boldsymbol{m} \times 1}$.

Plug the contact force $f_{\text {tip }}=f_{T}+N \boldsymbol{X}$ into the formula above to get the constraints with vector $\chi$

$$
A N \chi \leq B-A f_{T}
$$

and obtain the optimization model of vector $\chi$ based on the optimized target of Euclidean norm.

$$
\left\{\begin{array}{l}
\min \frac{1}{2} \chi \chi^{T} \\
\text { s.t. } \quad \boldsymbol{A N} \chi \leq B-\boldsymbol{A} \boldsymbol{f}_{T}
\end{array}\right.
$$

\section{The Analysis of the Steady for Grasping Explosive.}

1) The Analysis of the Steady under the Static Force

When neglecting the explosion fatalness of the grasping object, the larger of the nornal force the larger of the grasping explosive is ${ }^{[10-12]}$. The relation between the normal force of the finger and the weight of the explosive is shown in Fig 5.

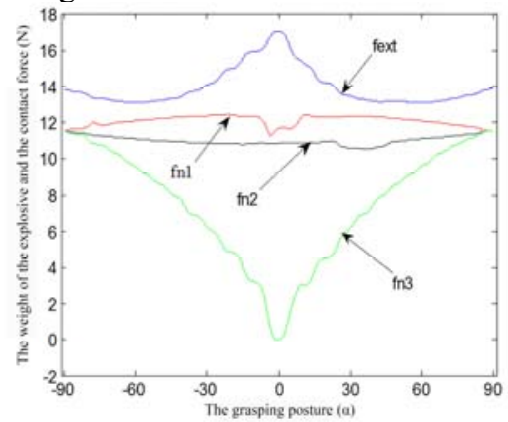

Figure 5. The relation between the normal force of each finger and the maximal weight of the explosive

However, as the analysis above, if too much normal force is added in the explosive or the dangerous objects in the operation, it will damage the explosive and even cause explosion $^{[13]}$. Therefore, in order to ensure the safety in grasping, it has to limit the normal force.

The weight of the explosive under the condition of the limited maximal normal force $\left(f_{\mathrm{n}}=10 \mathrm{~N}\right)$ is shown in Fig 6 . When the grasping weight ranges from $11.5 \mathrm{~N}$ to $16.6 \mathrm{~N}$, the grasping normal force of the fingers is no more than $10 \mathrm{~N}$ within the grasping range and it can meet the grasping limitation. So it can not only guarantee the operational reliability but also the secure operating of explosives for a certain degree.

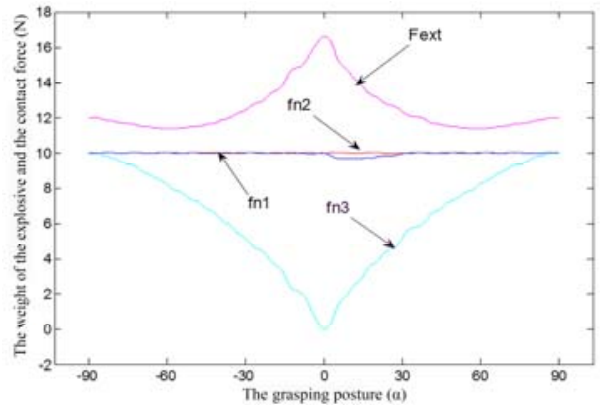

Figure 6 . The possible grasping weight when the maximal contact force is $10 \mathrm{~N}$

2) The Analysis of the Steady under the Impact Load Solving the Equation (8), while impacting the explosive, the impacting direction depends on the most negative posture of the grasping ability with the fingers ${ }^{[14]}$, i.e. when the grasping directions are $\alpha=30^{\circ}$ and $\beta=0^{\circ}$, the grasping weight of explosive is shown in Fig 6 that $m g=$ $11.5 \mathrm{~N}$. So it can get the coefficient matrix $\boldsymbol{A}$ and constant matrix $\boldsymbol{B}$. the zero-space matrix $\boldsymbol{N}$ can be got by the numerical method and now $\chi=\left[\begin{array}{lll}\chi_{1} & \chi_{2} & \chi_{3}\end{array}\right]$.

Using the output signal $\chi=\left[\begin{array}{lll}\chi_{1} & \chi_{2} & \chi_{3}\end{array}\right]$ and solving the problem above by the method of neural network, it gets the dynamic change process of the minimal normal force $\left(f_{\mathrm{n}}\right)$ of the three fingers under the impact load, which is illustrated in Figure 7.

The normal force of the finger changes under the impact force and when the vibration of the robot levels off, all the contact forces of the fingers tends to be steady ${ }^{[15]}$. It need the largest normal force while it's impacting, also it is the minimal normal force for the steady grasping, which is $12.85 \mathrm{~N}$.

By contrast, it is the maximal normal force $f_{\mathrm{n}}=10 \mathrm{~N}$ for grasping the same weight, which is $11.5 \mathrm{~N}$ of the explosive shown in Fig 6. Obviously, the required minimal normal force of the explosive is more than the normal force provided by the multi-fingered hand so the explosive is unstable, which is shown in Table 1.

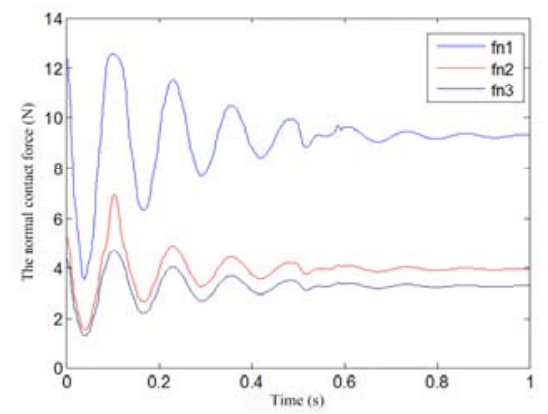

Figure 7. The dynamic response curves of minimal contact force 
TABLE 1 THE PARAMETER OF THE UNSTABLE EXPLOSIVES

\begin{tabular}{|c|c|c|c|c|}
\hline $\begin{array}{c}\text { The } \\
\text { parameter }\end{array}$ & $\begin{array}{c}\text { The posture } \\
\text { of the robot }\end{array}$ & $\begin{array}{c}\text { The } \\
\text { weight } \\
\text { of the } \\
\text { explosive }\end{array}$ & $\begin{array}{c}\text { The } \\
\text { maximal } \\
\text { normal } \\
\text { force } \\
\text { under the } \\
\text { static load }\end{array}$ & $\begin{array}{c}\text { The } \\
\text { required } \\
\text { minimal } \\
\text { normal } \\
\text { force under } \\
\text { the impact } \\
\text { load }\end{array}$ \\
\hline The value & $A=30^{\circ}, \beta=0$ & $11.5(\mathrm{~N})$ & $10(\mathrm{~N})$ & $12.85(\mathrm{~N})$ \\
\hline
\end{tabular}

\section{CONCLUSION}

This paper focus on the study of the ability for grasping the explosive and the stability of the explosive influenced by the impact load, then it can draw the conclusion in below.

(1) The ability of the explosive disposal robot for grasping the explosive has something to do with the posture of the robot. When the contact position between the finger and the explosive is given, the heaviest explosive for possibly grasping with multi-fingered hands takes place in $\left\{\begin{array}{l}\alpha=0 \\ \beta=60^{\circ}\end{array},\left\{\begin{array}{l}\alpha=0 \\ \beta=180^{\circ}\end{array}\right.\right.$ and $\left\{\begin{array}{l}\alpha=0 \\ \beta=300^{\circ}\end{array}\right.$, i.e. the external force direction is located in the middle between the two fingers, the grasping ability is best.

(2) Because the posture of the explosive disposal robot is changing when transporting the explosive, it should choose the minimum value of the optimization algorithm above as considering the stable grasping in any posture and position. The minimum value occurs in $\left\{\begin{array}{l}\alpha=-30^{\circ} \\ \beta=0^{\circ}\end{array},\left\{\begin{array}{l}\alpha=-30^{\circ} \\ \beta=120^{\circ}\end{array},\left\{\begin{array}{l}\alpha=30^{\circ} \\ \beta=0^{\circ}\end{array}\right.\right.\right.$ and $\left\{\begin{array}{l}\alpha=30^{\circ} \\ \beta=120^{\circ}\end{array}\right.$.

(3) Having considering the fatalness of the explosion, the paper has put forward the grasping ability under the condition of the limited normal force. It studies on the grasping stability of the explosive under the static load and dynamic load, obtains the minimum normal contact force for stable grasping by the method of screw theory and neural network to prevent the explosive getting off caused by the inadequate contact force, and analyzes the stability of explosives. The study of the paper has universal significance to determine the grasping force of the dangerous object.

\section{REFERENCES}

[1] Richard R.M, Zexiang Li, A Mathmatical Introduction to Robotic Manipulation[M], CRC Press, Inc., 1994

[2] Guo Yu Sun Zhijun. Multi-objective optimization grasping planning for multifingered robot hand[J], Journal of Southeast University ( Natural Science Edition), 2012,42(4): 643-648

[3] Chun-Hsu Ko, Shih-Hsiung Lin, and Jing-Kun Chen. Motion Planning of Multifingered Hand-Arm System with Optimal Grasping Force[J], IEEE 2nd International Symposium on Next-Generation Electronics, 2013, 262-265

[4] Vincenzo Lippiello, Bruno Siciliano, Luigi Villani, A Grasping Force Optimization Algorithm for Multiarm Robots With Multifingered Hands[J], IEEE Transactions on Robotics, 2013, 29(1):55 - 67

[5] Takayoshi YAMADA, Manabu YAMADA, Stability Analysis of Multiple Objects Grasped by Multifingered Hands with Revolute Joints in $2 \mathrm{D}[\mathrm{J}]$,International Conference on Mechatronics and Automation,2012,29(1): 1785 - 1792

[6] Qiang Li, Robert Haschke and Helge Ritter. Rotary Surface Object Manipulation by Multifingered Robot Hand[J], Proceedings of Robotics,2012;1-6

[7] Zichen Zhang, Gu, J. Grasp planning of 3D objects using genetic algorithm[J], IEEE International Conference on Automation and Logistics, 2012:646 - 651

[8] Ko, Chun-Hsu; Chen, Jein-Shan.Optimal Grasping Manipulation for Multifingered Robots Using Semismooth Newton Method[J], Mathematical Problems in Engineering,2013:1-10

[9] Mo Haijun, Study on Ability of Multifingered Robot Hands Grasping an Explosive[D], Guangzhou, 2008

[10] T Yoshikawa, Manipulability of Robotic Mechanisms[J], The International Journal of Robotics Research,1985,4(2): 3-9

[11] Xiong Caihua Xiong Youlun. Configuration Planning of Multifingered Grasp and Its Stability Index[J], Journal of Jianghan Petroleum Institute,1997 19(1): 85-88

[12] Guanfeng Liu, Zexiang Li. Real-Time Grasping-Force Optimization for Multifingered Manipulation Theory and Experiments[J], IEEE Transactions on Mechatronics, 2004 ,9(1) :65-76

[13] Akio Namiki ,Masatoshi Ishikawa, The Analysis of High-speed Catching with a Multifingered Robot Hand[J], IEEE International Conference on Robotics and Automation,2005,2666-2671

[14] Haidacher. S, Hirzinger. G. Contact Point Identification in Multi-Fingered Grasps Exploiting Kinematic Constraints[J], International Conference on Robotics and Automation , 2002, $1597-1603$

[15] Zhou Kaili,Kang Yaohong. Neural network model and MATLAB simulation programming, Beijing: Tsinghua University Press,2005 